\title{
Massive infarction of the uterus and appendages caused by torsion
}

\author{
E. DAW \\ N. SAleh \\ M.R.C.O.G. \\ M.R.C.O.G.
}

North Manchester General Hospital, Crumpsall, Manchester

\begin{abstract}
Summary
A case of torsion of the uterus and appendages is reported and discussed.

\section{Introduction}

A minor degree of rotation of the uterus is common but insignificant, especially in pregnancy, and is not sufficient to obstruct the blood supply in the normal uterus (Jeffcote, 1972). A patient is described who had massive infarction of an enlarged non-pregnant uterus and both appendages caused by torsion.

\section{Case history}

This obese patient was born in 1900 and was nulliparous. In 1942 she had been told that she had a 'large fibroid uterus' but was not fit for surgery. She had an uneventful menopause when aged 50 and a severe coronary thrombosis when 75 years old. She had been given anti-coagulants for 6 weeks
\end{abstract}

following this but had received no further treatment for her hypertension. In October 1978, she had a sharp pain in her right side whilst bending down. Straightening up had not relieved the pain, although it was eased slightly after she had vomited. She did not faint and the pain in her right side persisted. She was seen 3 days later, when a large tender, but mobile, mass in her abdomen arising from the pelvis was palpable. Torsion of a fibroid or fibroids was suspected and the patient admitted to hospital. At laparotomy under epidural anaesthesia it was found that the whole uterus, both tubes and ovaries had undergone torsion through 540 degrees. The uterus was distorted by fibroids and measured $20 \times 24 \times 10 \mathrm{~cm}$. All the twisted structures were infarcted and gangrenous (Fig. 1) and it was considered surprising that the patient had survived this episode. A hysterectomy and bilateral salpingo-oöphorectomy were performed. The patient started fibrillating and

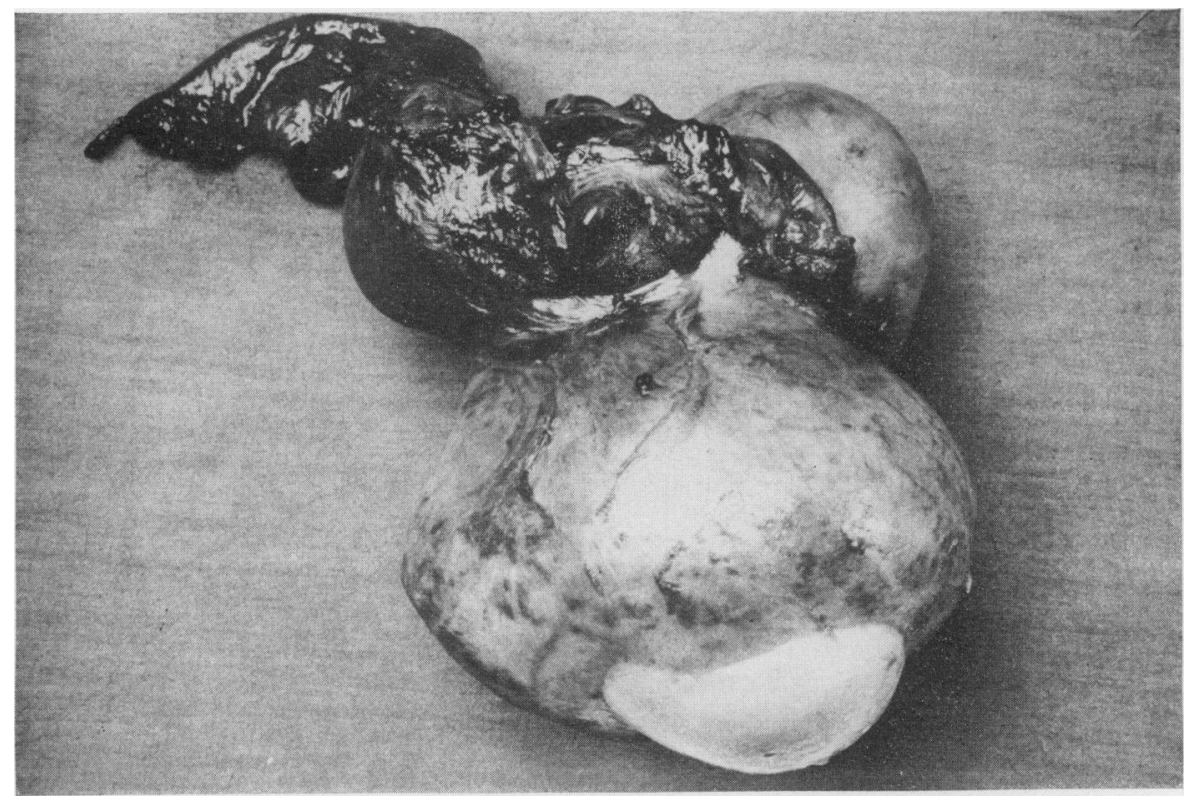

FIG. 1. The infarcted uterus and appendages above the large fibroids. 
had a cardiac arrest during the operation. Her heart restarted immediately resuscitation was commenced. In view of this she was nursed in the Intensive Care Unit for $36 \mathrm{hr}$, during which time she was digitalized. Apart from this, her postoperative progress was excellent and she was discharged home on the 9th postoperative day. Histological examination of the specimen removed at operation showed the infarcted leiomyoma to have spotty calcification and areas of hyaline degeneration. Infarction of the other structures was noted.

\section{Discussion}

Uterine torsion during pregnancy has been reported in over 100 cases (Piot, Gluck and Oxorn, 1973; Koh and Bradford, 1977). Torsion of the nongravid uterus has not been reported frequently (Bolognese, Weber and Zachary, 1967) and torsion of the uterus causing infarction of a fibromyoma even less so (Black and McFarlane, 1959). This infrequency is probably because the outcome is less tragic than in torsion of the gravid uterus, rather than infrequency of occurrence. Such massive infarction by torsion of the uterus has not been reported before and prompts the authors to report $\$$ this case.

The case is also unusual in that rotation through $540^{\circ}$ had occurred (i.e. 3 twists). A search of the $\stackrel{5}{\rightarrow}$ literature reveals only one case with such rotation and necrosis (Van Pall in 1940-reported by Piot 흠 et al., 1973).

\section{Acknowledgements}

We wish to thank Mrs M. Tudor for typing the manuscript and Miss J. Perry for the figure.

\section{References}

BlaCK, E.F.E. \& MCFARLANE, C.J. (1959) Torsion of the uterus causing infarction of a fibromyoma. Americanco Journal of Obstetrics and Gynecology, 77, 513.

Bolognese, R.J., Weber, L.L. \& ZaChary, T.V. (1967) cं Torsion of the nongravid uterus. Journal of the American? Medical Association, 199, 1011.

JeffCote, T.N.A. (1972) Principles of Gynaecology, 3rd Edn, $\stackrel{\oplus}{\oplus}$ p. 360 . Butterworths, London.

KoH, K.S. \& BRADFORD, C.R. (1977) Uterine torsion during pregnancy. Canadian Medical Association Journal, 117, 501. 음

PIOT, D., Gluck, M. \& OxORN, H. (1973) Torion of the gravid uterus. Canadian Medical Association Journal, $\rightarrow$ $109,1010$. 